\title{
IL BILINGUISMO TARDIVO: IL CASO DELLE "BADANTI"
}

\author{
Ana Benčić, Rita Scotti Jurić \\ Università Juraj Dobrila \\ Dipartimento di studi in lingua italiana
}

\section{Riassunto}

Se gli esseri umani sono "programmati" per l'acquisizione di una lingua, possiamo dedurre che siamo programmati anche per acquisirne più di una. È risaputo che l'età in cui viene introdotta la seconda lingua ha effetti importanti sullo sviluppo e sul funzionamento del cervello, sul modo in cui questo gestisce gli stimoli linguistici e semiotici. Sull'assunto che ipotizza la possibilità per tutti di diventare facilmente bilingui, la presente ricerca cercherà di constatare il livello di apprendimento di una lingua in età adulta. Si analizzerà il linguaggio di sei parlanti bilingui in età adulta, badanti presso persone anziane in Italia. Di seguito si spiegheranno le difficoltà linguistiche che i soggetti incontrano e il grado di bilinguismo raggiunto con alcune riflessioni comparative e conclusive.

Parole chiave: bilinguismo tardivo, apprendimento spontaneo, linguistica, pragmalinguistica.

\section{Alcune teorie sul bilinguismo}

Il significato del termine "bilingue" è diverso a seconda della situazione, dell'età, delle prospettive, delle motivazioni per cui le definizioni si muovono tra una concezione larga, ma pur realistica, e una concezione rigorosa e ristretta con pochi riscontri nella realtà. MacNamara (1967), dichiara bilingue chiunque parli più di una lingua, mentre Bloomfield (1993) proclama bilingue solo chi parla perfettamente due lingue. Secondo Titone (1972) le due lingue devono godere di una autonomia strutturale completa, senza sfruttamento dell'una nella costruzione di strutture o prodotti dell'altra «aderendo fedelmente ai concetti e alle strutture che a tale lingua sono propri, anziché parafrasando la lingua nativa» $(1972,13)$. Ambedue le definizioni mettono l'accento sul grado di competenza, e squalificano subito i parlanti che non possiedono la competenza linguistica richiesta. Per tale motivo, le definizioni che prenderemo come punto di partenza sono quella di Weinreich («Chiamerò qui bilinguismo la pratica di usare alternativamente due lingue, e bilingui le persone interessate», 1974, 3) e quella di Haugen («the point where a speaker can first produce complete meaningful utterances in the other language», 1953, 7) che pongono come criterio fondamentale l'uso e la necessità comunicativa e lasciano aperta la questione del grado di competenza linguistica. 
Il criterio che considera l'età di acquisizione della seconda lingua si pone come una barriera importante tra tipi differenti di apprendimento, distinguendo un bilinguismo dei bambini da quello degli adulti. L'approccio psicolinguistico al bilinguismo rivela il ruolo che ciascuna lingua assume in diversi periodi della vita di un individuo bilingue e spiega l'equilibrio dinamico che si stabilisce fra i due sistemi linguistici. Dal punto di vista del parlante è raro che i diversi sistemi di cui dispone si pongano sullo stesso piano, per cui non ha lo stesso grado di competenza linguistica nelle due lingue né attribuisce ad esse la medesima funzione. Weinreich (1953) è stato il primo a codificare la competenza bilingue come: coordinata, composita e subordinata. Più tardi Ervin e Osgood (1954) inseriranno il bilinguismo composito e quello subordinato in uno stesso gruppo, offrendo l'antinomia tra bilinguismo composito vs. coordinato. In base alla tipologia del bilinguismo composito il bilingue dispone di una struttura cognitiva unitaria, ovvero, pur facendo ricorso a espressioni distinte per designare un determinato referente nelle due lingue, ad esse fa corrispondere una stessa concettualizzazione. Si presuppone perciò che la persona abbia imparato le due lingue nello stesso contesto, per cui sono usate in concorrenza con una rappresentazione fusa delle stesse. Il bilinguismo coordinato implica che il soggetto disponga di due sistemi linguistici indipendenti: non solo i significati ma anche le unità di contenuto restano separati in maniera tale che in ciascuna lingua il parlante si costruisce un'autonoma serie di corrispondenze tra significante e significato. La persona avrebbe imparato le due lingue in ambienti separati, motivo per cui tende a mantenere separati i segni dei due idiomi. Il bilinguismo subordinato si realizza in fase di apprendimento quando la concettualizzazione nella L2 viene in qualche modo guidata dalle immagini mentali della L1. Questo tipo di bilinguismo si ha nelle situazioni in cui una lingua è chiaramente dominante sull'altra. A due significanti corrisponde un solo significato, al quale la lingua dominata può accedere solo attraverso la lingua dominante. Anche Ervin e Osgood (1954) ritengono che il bilinguismo coordinato si sviluppi come risultato dell'esperienza linguistica in vari contesti in cui le lingue incluse raramente si scambiano reciprocamente, a differenza del bilinguismo composito che si sviluppa in contesi linguistici dove continuamente avviene la commutazione del codice.

Chiariremo, per motivi di interesse terminologico di questa ricerca, i concetti bilinguismo bilanciato e dominante, bilinguismo additivo e sottrattivo. Il bilinguismo bilanciato presuppone una conoscenza pressappoco uguale, buona o cattiva delle due lingue, mentre quello dominante una conoscenza molto forte di una delle due lingue. Parliamo invece di bilinguismo additivo quando le capacità acquisite nella L2 si aggiungono a quelle della L1 senza però intaccarla; il bilinguismo sottrattivo avviene quando c'è una graduale sostituzione della prima lingua da parte della seconda.

I rapporti tra le lingue nel repertorio comunicativo di un bilingue sono dinamici e dipendono da diversi aspetti psicologici e sociali che determinano i modi d'uso della lingua e le sue funzioni, per cui non si esauriscono nelle classificazioni elencate (Grosejan, 1982). 


\section{Il bilinguismo tardivo}

Gli studi disponibili che trattano la problematica del bilinguismo tardivo sono pochi e difficilmente reperibili. Si tratta per lo più di brevi accenni, carenti di teorie consolidate o ricerche attendibili. Nel territorio istroquarnerino dove la problematica del bilinguismo adulto e tardivo è molto sentita, non ci sono studi in merito. Dalle poche fonti europee e americane rintracciate risulta chiaro che il bilinguismo tardivo viene definito come l'apprendimento della seconda lingua dopo la pubertà. Per Haugen (1953) esistono il child bilingualism e il late bilingualism, ossia la distinzione tra il bilinguismo dell'adolescenza e quello adulto. (Moretti-Antonini, 2000; Guasti, 2007).

\subsection{Acquisizione o apprendimento: la questione dell'età}

L'acquisizione di una lingua viene effettuata con modalità naturali, in un ambiente naturale, informale, con il coinvolgimento soprattutto della memoria implicita, a livello subconscio (Krashen, 1985). L'acquisizione per Krashen è profonda, stabile, e genera comprensione e produzione linguistica con processi automatici mentre l'apprendimento, razionale e volontario, è di durata relativamente breve e funge da monitor per l'esecuzione linguistica. Riguardo alla possibilità che l'apprendimento razionale possa trasformarsi in acquisizione, Krashen nutre solidi dubbi. L'apprendimento si realizza con modalità formali, per regole, quindi a livello conscio e con un incremento delle conoscenze esplicite sulla lingua. I due processi sono distinguibili in quanto c'è un coinvolgimento di strutture cerebrali diverse. Quando un parlante si trova di fronte al compito di dare un giudizio relativo ad una frase che sente, si comporta in due modi: se il giudizio si basa su regole che è in grado di spiegare, abbiamo a che fare con l'apprendimento, se invece si basa sulle sue sensazioni, si tratterà di acquisizione. Mentre nell'acquisizione l'attenzione è posta sul significato, nell'apprendimento essa è concentrata maggiormente sulla struttura linguistica e sulla correttezza onde evitare gli errori.

Esiste una modalità intermedia chiamata apprendimento naturale o spontaneo (Moretti e Antonini, 2000; Fabbro, 1996) in cui oltre all'istruzione formale il soggetto acquisisce la lingua nella vita quotidiana. Questa modalità intermedia è quella che prevale nell'acquisizione della L2 da adulti ossia nel bilinguismo tardivo. Gli elementi linguistici insegnati nelle classi portano all'apprendimento della competenza linguistica, ma questo processo guidato, con selezioni e graduazioni del materiale linguistico, il più delle volte non dà i frutti desiderati. Per una più completa acquisizione della competanza comunicativa si ha bisogno di apprendimenti spontanei, fuori dalla classe, che guidino gli apprendenti lungo un percorso naturale di acquisizione della L2. Oggi appare scontata la strategia di armonizzare i processi naturali di apprendimento spontaneo e quelli didattici, tipici dei contesti di istruzione formale.

Gli studiosi non sono unanimi nel determinare l'età che contraddistingue il bilinguismo tardivo. Per la Guasti (2007), si ha un'acquisizione precoce se i soggetti hanno appreso la L2 entro la pubertà, e un'acquisizione tardiva se la L2 è stata appresa in età adulta. Penfield e Roberts dichiarano: «for the purposes of learning languages, the human brain becomes progressively stiff and rigid after the age of nine» $(1959,236)$ 
Più in là diranno: «when languages are taken up for the first time in the second decade of life, it is difficult to achieve a good result because it is unphysiological» $(1959,255)$. Lenneberg (1967) ipotizza che esista il periodo di maggiore disponibilità entro il quale deve aver luogo l'acquisizione almeno della prima lingua, il che si aggira intorno ai quattro anni. Il calo della disponibilità nell'acquisizione sembra essere molto più lento e pare che continui anche in età adulta, come cercheremo di dimostrare nel saggio. Dalla pubertà entrano in scena l'imbarazzo, l'identità, la mancanza di empatia, tutti fattori che influenzano apertamente una pratica spontanea della lingua. Swadesh osserva che: «quanto più adulta è una persona al momento in cui viene in contatto con una nuova lingua, tanto meno sarà probabile che egli ne acquisti la piena padronanza» $(1941,61)$.

\section{Metodologia e scopo della ricerca}

La pendolarità lavorativa dall'Istria croata verso l'Italia, sempre più frequente negli ultimi vent'anni, crea un fenomeno di emigrazione e contatti di confine particolarmente interessanti. Non solo la vicinanza geografica, ma anche quella civile e culturale stabilita attraverso la storia tra i due Paesi, ha portato parecchie signore di tutte le età a cercare una stabilità lavorativa e finanziaria. Nel territorio istroquarnerino, in tutto il Veneto e nella Venezia Giulia queste collaboratrici domestiche che lavorano come infermiere ed aiutanti presso persone anziane o invalidi, a domicilio, vengono chiamate comunemente con il nome di "badanti".

I soggetti sottoposti a ricerca (tab. 1) sono state sei signore di madrelingua croata e bosniaca $(M=53.5)$, tre nate e cresciute a Pola dove vivono tutt'ora, altre tre nate in Bosnia ma residenti a Pola da molti anni. Quattro hanno terminato la scuola media superiore e due anche la facoltà. Quattro sono disoccupate e due sono pensionate. Solo le tre signore istriane hanno studiato l'italiano nella scuola media superiore per quattro anni, mentre le tre signore bosniache non hanno mai avuto occasione di studiare o parlare l'italiano prima di lavorare in Italia. Tutte sostengono di aver avuto il loro primo «vero» contatto con la lingua italiana appena oltre confine. In questo periodo di permanenaza in Italia $(\mathrm{M}=12,1)$ parlano la lingua italiana nell'ambiente lavorativo e nell' ambiente sociale.

Tabella 1. Struttura del corpus

\begin{tabular}{|c|c|c|c|c|}
\hline Soggetto & Provenienza & Età & $\begin{array}{c}\text { Permanenza in Italia } \\
\text { in anni }\end{array}$ & $\begin{array}{c}\text { Anni di studio della } \\
\text { L it. }\end{array}$ \\
\hline 1 & I & 42 & 10 & 4 medie sup. \\
\hline 2 & I & 53 & 12 & 4 medie sup. \\
\hline 3 & I & 64 & 9 & $/$ \\
\hline 4 & B & 47 & 15 & $/$ \\
\hline 5 & B & 69 & 14 & $/$ \\
\hline 6 & B & 46 & 13 & \\
\hline
\end{tabular}


Lo strumento principale della ricerca è stato l'intervista semi-strutturata che prevedeva una traccia di argomenti da affrontare durante l'intervista stessa, procedendo dal generale allo specifico. Lo scopo della presente ricerca è quello di presentare e discutere i fenomeni linguistici che avvengono in momenti di contatto delle lingue nel bilingue tardivo e sfatare l'interpretazione erronea che li vede essenzialmente come il risultato di uno scarso bilinguismo, ossia come indici di debolezza di uno dei due sistemi linguistici. Il campo sul quale affineremo le nostre teorie è quello dell'analisi dell'interlingua, ossia la lingua dei parlanti "apprendenti", sperando sempre che queste soluzioni linguistiche siano temporanee e che abbiano ancora possibilità di trovare gli equivalenti espressivi appropriati nella L2.

\section{Analisi del corpus}

\subsection{Analisi linguistica}

L'incontro tra due lingue nel linguaggio dei bilingui tardivi genera interferenze linguistiche (Mackey, 1970) causate dalle differenze strutturali delle medesime, per cui il parlante cercherà di riportare nella seconda lingua elementi e strutture della prima. Anche se le interferenze lessicali, fonologiche e morfosintattiche sono in buona misura prevedibili a priori in base alle strutture stesse delle lingue in contatto (Weinreich, 1974), non si escludono le interferenze extralinguistiche che ruotano attorno ad una dimensione individuale, intrapsichica.

L'interferenza fonologica riguarda il modo in cui un parlante percepisce e produce i suoni della seconda lingua in rapporto alla lingua nativa. Il biligue tardivo è esposto più degli altri bilingui all'identificazione dei fonemi del sistema secondario come fonemi del sistema primario. Nel parlato delle badanti bosniache e istriane abbiamo individuato parecchi casi di ipodifferenziazione di fonemi (che è in leto [letto]; no avevo kontato [contatto]). L'iperdifferenziazione di fonemi invece comporta l'imposizione ai suoni del sistema secondario di distinzioni fonemiche tratte dal sistema primario (ma altro parlo sempre itaরano [italiano]; metto il copriletto roza [rosa]). Casi di reinterpretazione di distinzioni sono individuabili nei fonemi $/ \mathrm{kw} / \mathrm{e} / \mathrm{gw} / \mathrm{che}$ corrispondono al grafema $q$ o $g$ seguito dalla $u$ semiconsonantica: di regola quasi in tutte le occorrenze viene pronunciato come $/ k v /$ (sono rimasta senza lavoro $k v a$, [qua] a Pola; un poco sapevo la lingva [lingua] ma sono passati tanti anni da qvando [quando] ho finito la scuola; avevo qvarantacinqve [quarantacinque], qvarantasei [quarantasei] anni).

La vera e propria sostituzione di foni si ha per quei fonemi che nelle due lingue hanno identica definizione ma diversa realizzazione. Così la $l$ italiana e' piuttosto alveolare, mentre quella croata è quasi dentale. Se poi a questo fenomeno assomiamo l'ipodifferenzazione o la reinterpretazione dei fonemi, alcune parole avranno il tipico accento croato (sono tutte bele [belle] persone, ben vestite, con i capeli beli [capelli belli], piturati; kveli [quelli] signori non parlano il dialetto).

Nell'interferenza morfosintattica troviamo un trasferimento di morfemi ossia una correlazione con la loro funzione grammaticale nella lingua d'origine e con la 
resistenza della lingua ricevente (ho finito ginnasia Branko Semelić a Pola; dopo due settimane posso tornarsi a casa). Vediamo in questi esempi che il morfema flessivo ricalca il genere femminile del sostantivo in lingua croata, mentre in italiano ginnasio è di genere maschile. Nel secondo esempio viene mantenuto il pronome riflessivo croato. Anche l'interferenza dell'ordine delle parole nella frase è molto frequente, accanto all'ommissione dell' articolo davanti ai sostantivi, articoli, determinativi, indeterminativi e partitivi in quanto la lingua croata non conosce gli articoli (questa rossa macchina; grammatica sapevo un poco ma mi mancavano le parole). Per quanto riguarda l'accordo e la dipendenza troviamo ausiliari sbagliati o l'ausiliare essere con un verbo transitivo scorretto (quando sono resto senza lavoro, sono cominciato pensare; È trovato la lavoro e in una casa di riposo e cominciato a fare lavori; piano, piano comiciato con giornali, con televisore).

Ulteriori interferenze troviamo nell'uso delle preposizioni che vengono letteralmente tradotte nella L2 o non vengono indicate (In mattina mi alzo, mi lavo; io non vado mai dormire $a$ pomeriggio la televisione; posso leggere normale nel giornale). Tutti i soggetti intervistati usano la forma estesa dell'aggettivo indefinito poco in enunciati in cui dovrebbe apparire la forma tronca po'per l'influenza del croato che non conosce questa dualità (Un poco forse con cibo; ma primi anni un poco avevo difficoltà).

Le interferenze lessicali rappresentano trasposizioni di modelli morfologici o sintattici dalla lingua d'origine a quella d'arrivo. Si tratta di prestiti non adattati, occasionali, come risultato della personale esperienza dell'altra lingua da parte dei singoli parlanti (Graffi, Scalise, 2002) (Quando c'è tempo guardo televisione, neke serije, che mi interessano).

\subsection{Analisi pragmalinguistica}

Le deviazioni dall'uso convenzionale non possono essere sempre spiegate come una conseguenza di una scarsa competenza grammaticale, ma come un'inadeguatezza sociopragmatica e socioaffettiva. Il problema più grande sta però nel fatto che i parlanti nativi, incoraggiati da una ormai classica visione della linguistica e della grammatica, attribuiscono, il più delle volte, le carenze linguistiche a tendenze caratteriali piuttosto che a fattori di competenza comunicativa (Barron, 2003), e fabbricano facilmente pregiudizi sulla personalità dell'interlocutore non-nativo (Bettoni, 2006). Mancando di un codice adeguato, le badanti croate, nel loro sforzo di comunicazione, quando devono affrontare problemi urgenti di comportamento con i loro badati, generano uno stile piuttosto direttivo. Il linguaggio dei sei soggetti denota una visiva incapacità di attenuare l'intensità dei loro atti e si serve di una gamma di mitigatori piuttosto ristretta se paragonata con quella dei parlanti nativi. Questa inadeguatezza pragmatica compromette la loro efficacia comunicativa e tende a generare nell'interlocutore nativo una falsa impressione di scortesia, in particolare negli atti di richiesta e di protesta (Signora, tu devi mangiare, brodo se fredda; Mettere un pullover, signora, fuori freddo; Lei si tiene qui, prego). Gli esempi riportati rivelano che i soggetti, anche se quotidianamente a contatto con la lingua obiettivo, faticano a raggiungere un comportamento adeguato dal punto di vista pragmalinguistico. Questo tipo di parlato, chiamato foreigner talk, è 
dovuto anche al fatto che la lingua sentita attorno a loro è spesso semplificata e lo stile conversazionale è contraddistinto da un alto grado di direttezza ${ }^{1}$. Eppoi: nell'interazione il croatofono si aspetta delle chiavi contestuali più esplicite essendo abituato, nella sua cultura e civiltà, ad un linguaggio diretto, mentre l'italofono ritiene poco educato essere troppo diretti ed usa più contextualizers. Come altri avverbi e locuzioni avverbiali (ad es. per cortesia), anche prego è un marcatore di cortesia, deputato anch'esso ad attenuare l'impatto del direttivo. In secondo luogo, anche se la frase è direttiva, o addirittura autoritaria, la badante, pronunciandola, si deve essere accorta che un tale messaggio genera un significato impositivo per cui cercherà di attenuare questa scortesia o comando con un prego, inteso come una sorta di metacomunicazione, aggiunta alla comunicazione primaria (la parte precedente del messaggio) oppure come un intercalare abbinato alle frasi come semplice atto di gentilezza.

Il parlato delle "badanti" è fortemente contestuale, testimoniabile dall'uso delle forme deittiche, in particolare del deittico temporale dopo. Questa non è certo una strategia comunicativa nuova visto che, secondo alcune stime, almeno il $90 \%$ degli enunciati dichiarativi di una lingua ha a che fare con l'indessicalità, cioè con la deissi (Bar-Hillel, 1973). La parola dopo indica il periodo di tempo in cui avvengono varie azioni, ma colma anche le lacune linguistiche della parlante e viene inteso come stampella linguistica. Il discorso delle badanti non si sviluppa per subordinazione, ma per coordinazione, con l'uso di frasi paratattiche, in ciò simile al linguaggio infantile. Possiamo ipotizzare che le parlanti tardive di L2 riattivino la capacità innata ontogenetica nella gestione della lingua straniera (Dopo viene una signora che pulisce la casa e io vado fuori. E resto fuori due, tre ore. Dopo torno, faccio pranzo, dopo pranzo signora va dormire, di nuovo la lavo cambio pannolone. E dopo lei si riposa, posso riposare, riposarsi anch'io ma, io non vado mai dormire a pomeriggio).

Un'altra caratteristica del linguaggio esaminato è il code mixing ovvero la giustapposizione di elementi linguistici appartenenti a due codici diversi in uno stesso enunciato. La confluenza dei due sistemi linguistici avviene a livello intrafrasale, nello stesso contesto senza che ai segmenti inseriti sia possibile attribuire un qualunque valore funzionale. È spesso rappresentato da una singola parola all'interno della frase. Quindi esso non avviene per la decisione dei parlanti ma inconsciamente, per colmare le lacune linguistiche. Nei soggetti troviamo code mixing dalla lingua italiana (Treba ih, ovaj srediti, ustat, dignut iz kreveta, pre, ih pripremiti za dan, promijeniti im pannolone, prat ih), da quella croata (Quante volte mi va, mi zbriše, qualche parola, ma adesso, piano piano no) e dal dialetto istroveneto (E dopo, dopo bisogno con lori poco camminare, preparare pranzo, dare mangiare. Estate venivano, lavorato a Medulin, venivano stranieri, così questo momento ho imparato tante robe).

Siccome la commutazione di codice viene considerata uno strumento forte della strategia comunicativa del parlante biligue, è quasi inesistente nei bilingui tardivi,

1 Termine usato da Blum Kulka e colleghi (1989) nella definizione della scala di direttezza degli atti di richiesta, ma anche in Yli Jokipii ( 1994). Parizzi, F., parla di parametri di esplicitezza vs. implicitezza e direttezza vs. indirettezza, (pp. 92 e 203) in Comunicare al lavoro. Studio delle pratiche comunicative messe in atto all'interno di un'organizzazione aziendale italiana, con particolare riferimento all'impatto esercitato dalle nuove tecnologie, Tesi di dottorato, 2005. $\mathrm{http}: / /$ ww.google.hr/url? sa=t\&rct=j\&q=direttezza $\% 20$ pragmalinguistica\&source=web\&cd=1\&sqi=2\&ved=0CCkQFj AA\&url=http $\% 3 \mathrm{~A} \% 2 \mathrm{~F} \% 2 \mathrm{Fwww}$. humnet.unipi.it $\% 2 \mathrm{Fdott}$ linggensac\%2Fmateriale $\% 2 \mathrm{Ftesi}$ dottorato.parizzi.doc\&ei= Hp15UfuCMYyZhQfxoYCwCw\&usg=AFQjCNE7OXHiPWzkvIGk61_WKF6GPT11Jg\&bvm=bv.45645796,d.d2k 
essendo connesso piuttosto ad un livello sociale di bilinguismo, come strumento atto a conciliare le due identità culturali delle due lingue (Heller, 1988). Per i bilingui tardivi, la seconda lingua è solo uno strumento di comunicazione, privato di rapporti culturali.

\section{Conclusione}

Possedendo già una lingua e un'identità culturale, le badanti costruiscono una conoscenza nuova sulle conoscenze che già possiedono per cui il loro bilinguismo è dominante. Il linguaggio esaminato presenta, a livello linguistico, un alto numero di interferenze che si manifestano nelle situazioni di contatto e che possiamo distinguere come abbandono di distinzioni obbligatorie che si traduce nella scomparsa di categorie grammaticali. Gli intervistati si concentrano sul significato di quanto vogliono trasmettere, mentre gli enunciati sono spesso grammaticalmente scorretti in particolar modo la morfologia e la sintassi. Siamo dell'idea che nei parlanti di italiano L2, con un livello di competenza linguistica medio-bassa, avvenga una fossilizzazione nello sviluppo dell'interlingua, e che la suddetta coinvolga soprattutto la dimensione pragmatica. I soggetti non hanno saputo dare un giudizio di grammaticalità di alcune frasi in quanto la loro conoscenza dell'italiano si basa sull'addestramento dell'“orecchio". Esso punta prima di tutto al riconoscimento dei contesti in cui queste forme devono essere usate e alla memorizzazione di un repertorio di formule verbali ad alta frequenza nonché al suo utilizzo in tempo reale (Lupo in bocca, invece di In bocca al lupo). Dall'analisi linguistica del linguaggio registrato traspare un discorso dove le concettualizzazioni sono guidate dalle immagini mentali della madre lingua, il croato, che esibisce un'influenza forte sulle strutture delle frasi in italiano. Le interferenze più evidenti, chiamate "indicatori del bilinguismo tardivo" sono quelle fonologiche che determinano una percezione dei suoni diversa da quella della lingua madre, per cui l'italiano rimarrà per sempre lingua straniera, non sentita come propria. Il livello di padronanza della lingua dei soggetti istriani si può definire B1, ossia livello autonomo soglia, mentre per quelli bosniaci la padronanza della lingua italiana è A2, cioè livello base intermedio o di sopravvivenza. Nel caso dei soggetti istriani la competenza in lingua italiana pur essendo molto scarsa, i residui di una scuola superiore, dopo una decina di anni di lavoro in Italia è migliore di quella dei soggetti bosniaci che vi lavorano da più di quindici anni, ma continuano a persistere problemi linguistici a tutti i livelli della lingua, con interferenze fonologiche, morfosintattiche e lessicali. Il fattore fondamentale in base al quale si differenziano le competenze delle signore istriane e bosniache è prima di tutto il tipo di acquisizione. Anche se potrebbe sembrare un fatto trascurabile, lo studio dell'italiano nella scuola superiore dei soggetti istriani ha fatto sì che l'acquisizione della morfosintassi della lingua italiana in tarda età fosse più soddisfacente, con una maggiore coesione e coerenza negli enunciati. L'esclusivo contatto con la lingua italiana nell'ambiente sociale, che conosce scambi linguistici esclusivamente in istroveneto, senza insegnamenti formali, non poteva offrire ai soggetti bosniaci buoni esempi di grammaticalità e funzionalismo della struttura per cui si risente della discontinuità delle frasi e della carenza lessicale.

In campo pragmalinguistico tutti gli intervistati presentano un parlato fortemente deittico con atti linguistici direttivi, senza tentativi di indirettezza e con scarsa volontà di 
modulare la forza illocutoria. Nelle interazioni con parlanti nativi, e in particolare nella formulazione di richieste, i soggetti bosniaci adottano un comportamento linguistico poco appropriato per quel che concerne l'uso degli strumenti di mitigazione. Possiamo comunque concludere che il parlante L2 tardivo, pur possedendo un codice molto povero, si accorge del tono e del significato emotivo di quanto sta dicendo per cui tenta, sul momento, di limitare il tono impositivo e di restituire alla frase un più corretto senso di cortesia. Ci sarebbe insomma, da parte del parlante, una sorta di autocontrollo immediato del valore e del significato dei messaggi pronunciati.

A prescindere dai dati ottenuti, siamo dell'opinione che sia doveroso attivare l'interesse per lo studio del bilinguismo. In una società che fa del long life learning un principio di vita si dovrà tenere in seria considerazione l'opportunità di trovare nuove soluzioni didattiche a tale scopo.

\section{BIBLIOGRAFIA}

BAR-HILLEL, Y., 1954, Indexical Expressions, in Mind, 63, pp. 359-379, (trad. it.) 1973, Espressioni indessicali, in BONOMI, A., (a cura di), La struttura logica del linguaggio, Milano, Bompiani, pp. 455-477.

BARRON, A., 2003, Acquisition in Interlanguage Pragmatics. Learning how to dothings with words in a study abroad context, Benjamins, Amsterdam-Philadelphia.

BETTONI, C., 2006, Usare un'altra lingua. Guida alla pragmatica interculturale, Laterza, Roma - Bari.

BLOOMFIELD, L., 1933, Language, Holt, Rinehart and Winston, New York.

BLUM-KULKA, S., 1989, Playing it Safe: The Role of Conventionality in Indirectness, in BLUM-KULKA, S., HOUSE, J., KASPER, G. (a cura di), Cross Cultural Pragmatics: Requests and Apologies. Nordwood, Ablex Publishing Corporation New Jersey.

ERVIN, S., OSGOOD, C. H., 1954, Second language learning and bilingualism, in «Journal of Abnormal and Social Psychology Supplement».

FABBRO, F., 1996, Il cervello bilingue. Neurolinguistica e poliglossia, Astrolabio, Roma.

GRAFFI, G., SCALISE, S., 2002, Le lingue e il linguaggio. Introduzione alla linguistica, Il Mulino, Bologna.

GROSEJAN, F., 1982, Life with two languages, Cambridge/Mass, Harvard U.P.

GUASTI, M. T., 2007, L'acquisizione del linguaggio. Un'introduzione, Raffaello Cortina, Milano.

HAUGEN, E., 1953, The Norwegian language, in America: A study in bilingual behaviour, Bloomington, Indiana U.P.

HELLER, M., 1988, Codeswitching. Anthropological and sociolinguistic perspectives, Mouton de Gruyter, Berlin.

KRASHEN, S., 1985, The Input hypothesis: Issues and implications, Longman, London.

LAUDANNA, A., BURANI, C., 1993, (a cura di), Il lessico: procedimenti e rappresentazioni, La Nuova Italia scientifica, Roma. 
LENNEBERG, E., 1967, Biological foundations of language, Wiley and Sons, New York.

MACKEY, W. F., 1970, The description of bilingualism, in FISHMAN, J., Readings in the Sociology of Language, Mouton, The Hague.

MACNAMARA J., 1967, The bilingual's linguistic performance. A psychological overview, in «Journal of Social Issues» 23.

MORETTI, B., ANTONINI, F., 2000, Famiglie bilingui, Dadò, Locarno.

PENFIELD, W., ROBERTS, L., 1959, Speech and brain mechanism, Princeton U.P., Princeton.

TITONE, R., 1972, Bilinguismo precoce ed educazione bilingue, Armando, Roma.

WEINREICH, U., 1974, Lingue in contatto, Boringhieri, Torino. (trad. dell'originale del 1953).

YLI-JOKIPII, H. M., 1994, Requests in Professional Discourse: A CrossCultural Study of British, American and Finnish Business Writing, Suomalainen Tiedeakatemia, Helsinki. 


\section{KASNA DVOJEZIČNOST: SLUČAJ NJEGOVATELJICA}

Istraživanje se bavi kasnom dvojezičnošću koja se utvđuje na temelju dobi stjecanja drugoga jezika. Sagledavajući problem dvojezičnosti odraslih govornika razjasnili smo razliku između pojmova stjecanje jezika i učenje jezika (Krashen, 1985). Time je otvoreno pitanje kritične dobi što ukazuje da ne postoje fiziološke granice u stjecanju L2, ali postoje razdoblja života u kojima je čovjek više ili manje predodređen za ovladavane jezikom.

Opis lingvističkoga i pragmalingvističkoga profila istraženih subjekata doveo nas je do uočavanja bitnih karakteristika dvojezičnih govornika. Zaključili smo da je kasna dvojezičnost subordinantna, dominanta i adiktivna, što dovodi do jezičnih fenomena kao što su jezične interferencije i code-switching, o čemu smo izvjestili u radu. Važnost istraživanja kasne dvojezičnosti osobito je izražena na području Istarske županije, pogotovo uoči uključenja Hrvatske i Istre u Europsku Uniju.

Ključne riječi: kasna dvojezičnost, stjecanje jezika u prirodnim okolnostima, lingvistika, pragmalingvistika.

\section{LATE BILINGUALISM: CARER CASE STUDY}

This study examines the late bilingualism, based on the age of acquisition of the second language. Looking at the issue of adult bilingual speakers we clarified distinction between language acquisition and language learning (Krashen, 1985). The critical age is still an open issue, and we can conclude that there are no physiological limits in L2 acquisition. There are, however, periods of life in which man is more or less destined for learnining a language. The description of subjects' linguistic and pragmalinguistic profile, brings us to identify the essential characteristics of bilingual speakers. We conclude that the late bilingualism is subordinated, dominant and addictive, which consequently causes the linguistic phenomena such as linguistic interference and codeswitching, reported in the paper. The importance of research of late bilingualism is particularly strong in a bilingual area of Istria, especially on the eve of Croatia and Istria becoming part of the European Union.

Key words: late bilingualism, natural acquisition language, linguistic, pragmalinguistic. 